\title{
Paraganglioma del nervio vago
}

\author{
E. Torres-Carranza; P. Infante-Cossío; A. García-Perla; R. Belmonte; J. Menéndez y J.L. Gutiérrez-Pérez
}

Servicio de Cirugía Oral y Maxilofacial. Hospital Universitario Virgen del Rocío. Sevilla.

\section{Resumen}

Los paragangliomas del nervio vago son tumoraciones vasculares benignas muy infrecuentes, de origen neuroectodérmico. Se manifiestan clínicamente como masas cervicales asintomáticas, aunque a veces pueden provocar afectación de pares craneales. Rara vez se acompañan de niveles elevados de catecolaminas. El diagnóstico se basa en la clínica apoyada en pruebas de imagen, donde la TAC y la RNM juegan un papel importante. La angiografía no sólo permite el diagnóstico sino además la realización de una embolización preoperatoria. El tratamiento más aceptado es la extirpación quirúrgica. Otra opción terapéutica en función de la lesión y de la situación del paciente es la radioterapia. En este trabajo se presenta un nuevo caso de un paraganglioma del nervio vago de localización cervical, con clínica de crisis hipertensivas y niveles altos de catecolaminas. Se realiza una revisión de la literatura y se describen las formas de presentación clínica, el diagnóstico y el manejo terapéutico de esta rara entidad.

PALABRAS CLAVE: Glomus vagal. Paraganglioma. Funcionante.

\section{Paraganglioma of the vagus nerve}

\section{Abstract}

Paragangliomas of the vagus nerve are uncommon vascular benign neoplasms of neuroectodermic origin. Initial clinical manifestation is usually as an asymptomatic cervical mass, although sometimes may cause lower cranial nerve palsies. These paragangliomas seldom associate to high levels of circulating catecholamines. Diagnosis is based on the clinics aided by imaging, where CT and MRI play an important role. Angiography is not only diagnostic, but it also allows preoperative embolization of the mass. Most accepted treatment is surgical

Recibido: 31-03-05. Aceptado. 20-05-05 removal, even though some paragangliomas are suitable for radiation therapy in very specific patients. In this paper we describe a new case of paraganglioma of the vagus nerve in a cervical location, with hypertensive episodes and high catecholamine-levels. The authors review the literature describing the clinical presentation, the diagnosis and the treatment of this rare lesion.

KEY WORDS: Glomus vagale. Functioning. Paraganglioma.

\section{Introducción}

El paraganglioma del nervio vago o glomus vagal es una tumoración benigna vascular muy infrecuente y de origen neuroectodérmico ${ }^{7,19}$. Supone menos del 5\% de todos los paragangliomas de cabeza y cuello y de los que hay aproximadamente 200 casos publicados en la literatura ${ }^{17}$. En esta localización son los terceros en frecuencia, después de los del cuerpo carotídeo y los yugulotimpánicos ${ }^{19}$. Representan aproximadamente el 10\% de los tumores benignos que asientan en la fosa infratemporal ${ }^{19}$. Se estima que sólo del 1 al 3\% de los paragangliomas de cabeza y cuello secretan catecolaminas, a partir de los gránulos citoplásmicos neurosecretores que contienen, aunque la repercusión funcional de su neurosecreción es aún menor ${ }^{6}$.

El propósito de este trabajo es analizar un nuevo caso de un paciente afecto de paraganglioma del nervio vago, presentar nuestra experiencia en el diagnóstico y manejo terapéutico de esta rara entidad, y realizar una revisión de la literatura al respecto.

\section{Caso clínico}

Paciente varón de 59 años que acude a Urgencias para valoración por Otorrinolaringología por cuadro catarral y odinofagia de 4 meses de evolución. Como antecedentes personales de interés destacan: fumador desde hace 30 años de dos paquetes de cigarrillos al día, cefaleas frecuentes que ceden con analgésicos e hipertensión mal controlada, presentando frecuentes crisis hipertensivas con taquicardia, sofocos y sudoración abundante, a pesar de realizar tratamiento médico. No presenta signos neurológicos de afectación de pares craneales. A 


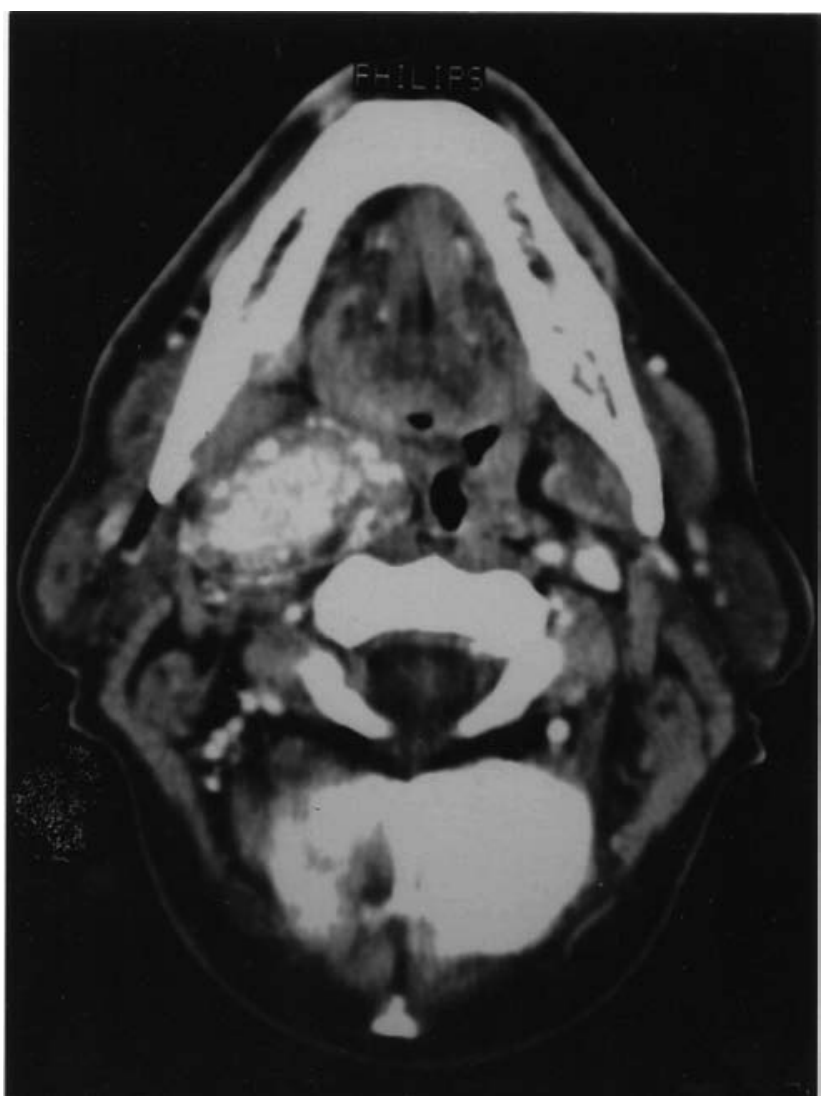

Figura 1. TAC con contraste donde se visualiza lesión de 3 $x 3.5 \times 6 \mathrm{~cm}$ en el espacio parafaríngeo y su relación con estructuras vecinas.

la exploración de la cavidad oral no se visualiza ninguna alteración patológica y en la faringe se objetiva la mucosa ligeramente eritematosa, especialmente en el lado derecho, con escasa mucosidad. Por palpación se aprecia una tumoración yugulodigástrica derecha de aproximadamente $5 \times 4 \mathrm{~cm}$ de tamaño, débilmente pulsátil, no dolorosa y de consistencia dura, compatible con una adenopatía cervical. Se realiza una laringoscopia indirecta visualizándose una tumoración que protuye en la faringe.

En la TC con contraste (Fig. 1) y en la RNM se visualiza una lesión ovalada única, altamente vascularizada, con márgenes bien delimitados, con realce homogéneo e intenso, que se encuentra en relación con el paquete vasculo-nervioso cervical. La lesión se extiende desde la base del cráneo partiendo del foramen yugular derecho, que aparece ensanchado respecto al contralateral, progresando inferiormente por el espacio parafaríngeo y protuyendo sobre la pared faríngea del lado derecho. Esta tumoración se sitúa postero-medial al paquete yugulo-carotídeo en los cortes más inferiores, desplazando ambas carótidas anteriormente, llegando hasta la altura del hueso hioides y en relación con el nervio vago. Las medidas son de $3 \mathrm{~cm}$ en el

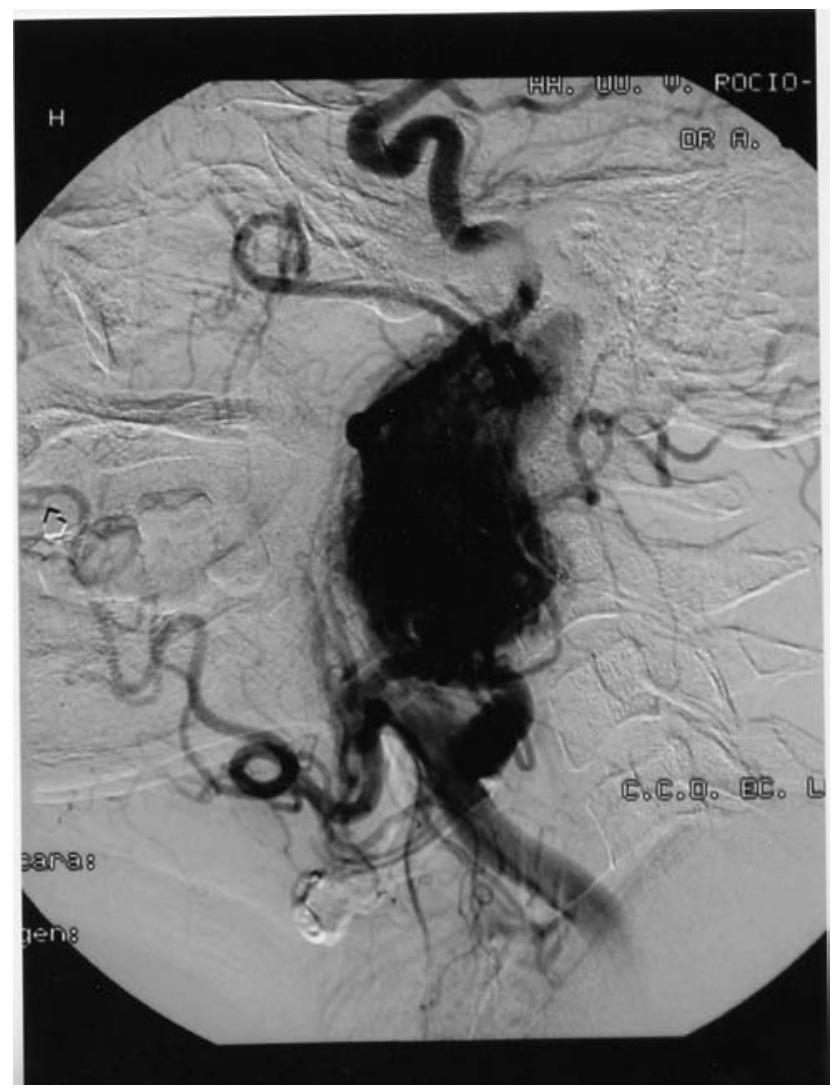

Figura 2. Angiografia donde se comprueba el desplazamiento anterior carotídeo y el teñido vascular.

eje anteroposterior, $3.5 \mathrm{~cm}$ en el transversal y $6 \mathrm{~cm}$ en el eje longitudinal. No se visualizan adenopatías de tamaño significativo en los espacios cervicales. Con el diagnóstico de presunción de glomus vagal se realizó un estudio de niveles de catecolaminas en orina de 24 horas encontrándose valores elevados de metanefrinas (95, valor normal $0-5$ ) con excreción de ácido vanilmandélico normal. El rastreo con metaiodobenzylguanidina muestra captación aumentada en la región cervical.

Dada la situación clínica del paciente y la resecabilidad de la tumoración se decidió la extirpación quirúrgica de la misma. Previamente, se realizó una angiografía (Fig. 2) en la que se observa que la vascularización tumoral proviene principalmente de la arteria faríngea ascendente, a través de la cual se embolizó un $98 \%$ de la lesión, dos días antes de la intervención quirúrgica. Se realiza bloqueo $\alpha$ y $\beta$ adrenérgico preoperatorio y la extirpación quirúrgica (Fig. 3) se realiza mediante un abordaje cervical anterolateral paralelo al esternocleidomastoideo por el que se accede al tumor, logrando preservar los nervios hipogloso, glosofaríngeo y el plexo cervical alto, así como las arterias carótida interna, externa y la vena yugular interna. Durante la intervención no presenta crisis de taquicardia. Tras la intervención se extuba y perma- 


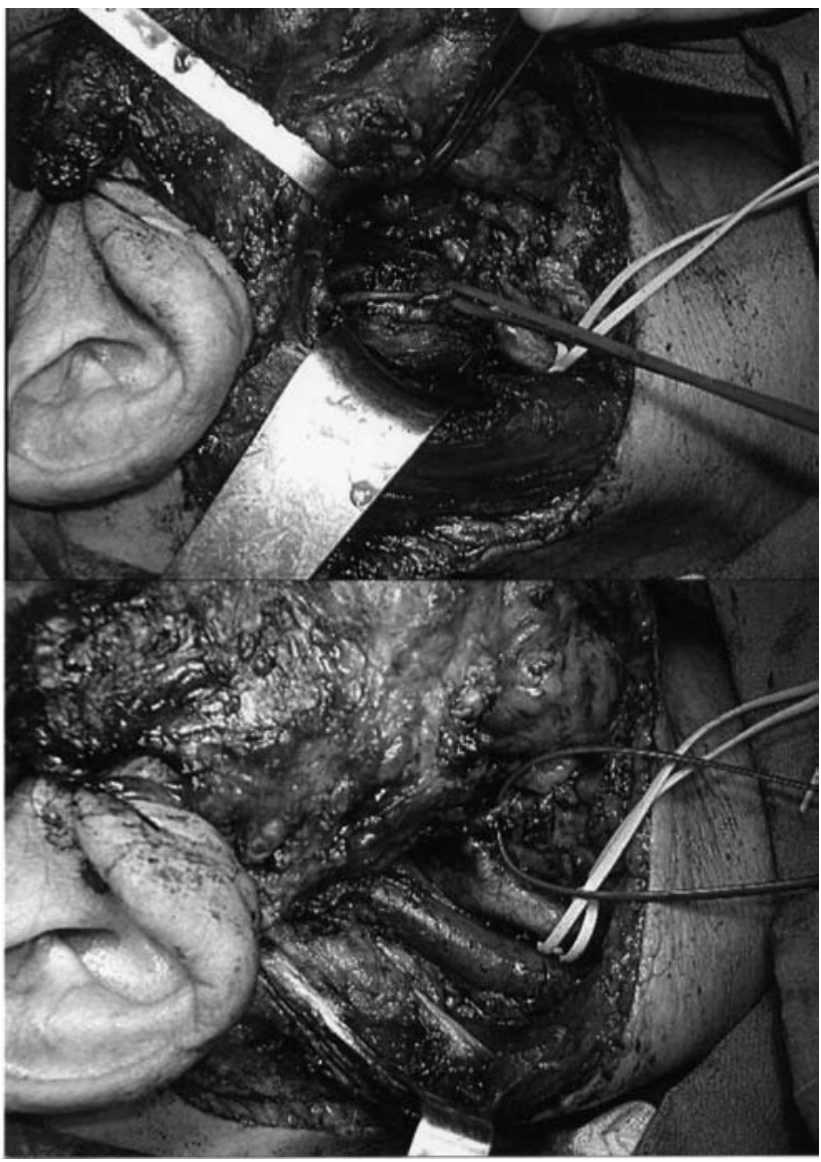

Figura 3. Visión de la tumoración procedente del vago. Relación con los grandes vasos cervicales una vez extirpada la tumoración.

nece las primeras 48 horas del postoperatorio en la Unidad de Cuidados Intensivos. Ni durante ni después de la cirugía han sido necesarias transfusiones sanguíneas, contabilizándose una pérdida hemática aproximada de $490 \mathrm{ml}$. El análisis anatomopatológico de la lesión extirpada confirma el diagnóstico preoperatorio de sospecha. Tras la extirpación de la lesión, con sacrificio del nervio vago, se ponen de manifiesto signos de afectación funcional del mismo. El paciente presentó disfonía por una parálisis de la cuerda vocal homolateral, que solucionó posteriormente, con reeducación de la voz por un logopeda. En los siguientes controles, los niveles de catecolaminas descendieron, y las cifras tensionales se controlaron con el tratamiento médico. En los tres años siguientes a la intervención no se han reproducido las crisis hipertensivas. El resultado estético es adecuado y no se observan signos ni síntomas de recurrencia de la enfermedad (Fig. 4).

\section{Discusión}

El paraganglioma del nervio vago es un tumor no cro-

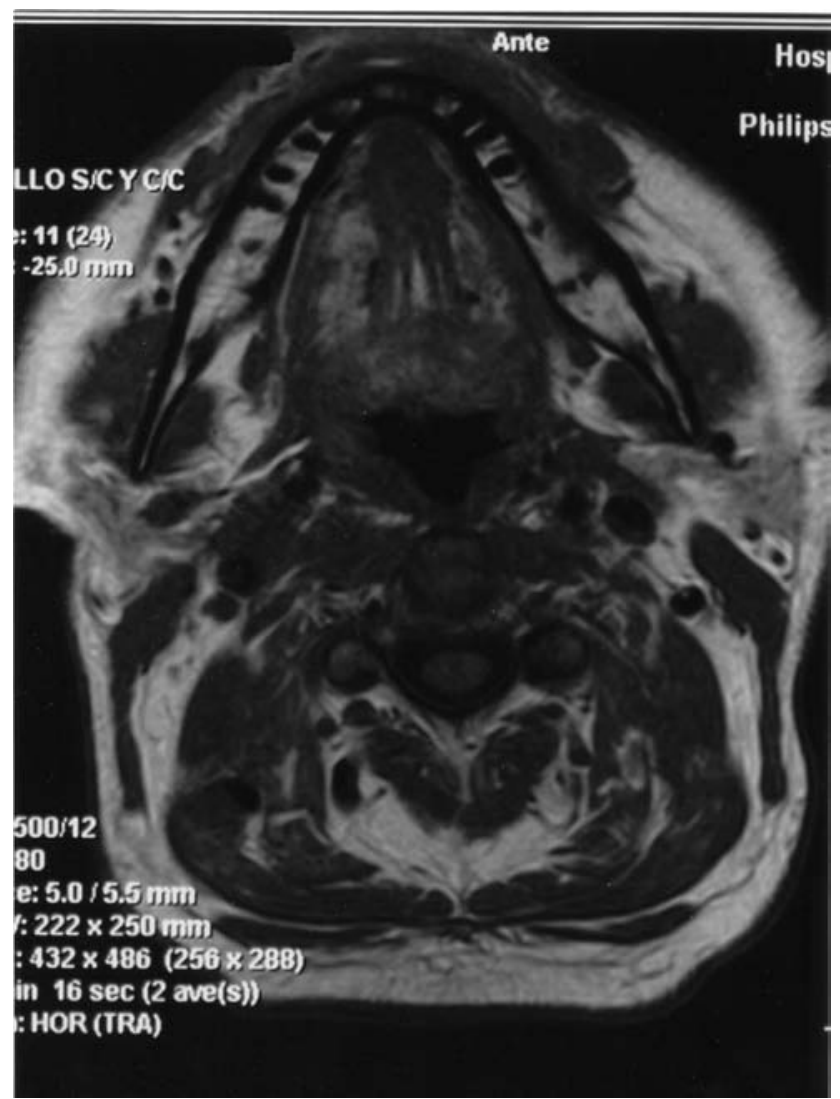

Fig. 4: RNM de control tras la extirpación del paraganglioma.

mafin, que surge predominantemente en los acúmulos de células paraganglionares dentro del perineurio del nervio vago, asociado a uno de los tres ganglios del mismo ${ }^{7,17,19}$. Principalmente, se asocia al ganglio más inferior, conocido como ganglio nodoso, que coincide con los dos primeros centímetros extracraneales del nervio ${ }^{17}$. Puede limitarse a la región cervical, unirse firmemente a la base de cráneo o extenderse intracranealmente ${ }^{17}$. Algunos autores creen que el ganglio de origen del glomus vagal puede determinar la forma de presentación clínica y el patrón de crecimiento del mismo, de tal manera que los situados en el ganglio nodoso tienden a permanecer en la región cervical mientras que los del ganglio medio alcanzan base de cráneo y los del ganglio superior pueden llegar a extenderse intracranealmente ${ }^{17}$.

La etiología de estas lesiones parece que está en relación con hipoxia tisular, ya sea por altitud elevada o por situaciones patológicas de baja oxigenación tisular, como EPOC o cardiopatías con bajos niveles de oxigenación de la hemoglobina ${ }^{19}$. En este paciente no encontramos antecedentes patológicos de este tipo. Se suele presentar con más frecuencia en la $5^{\mathrm{a}}-6^{\mathrm{a}}$ década, en el sexo femenino, a diferencia del caso que nos ocupa que se trata de un varón. Se trata de tumores de crecimiento lento, lo que con- 
lleva un retraso de media de tres años entre la aparición de los síntomas y el diagnóstico ${ }^{19}$, aunque en este paciente se llegó al diagnóstico a los cuatro meses de la aparición de las molestias faríngeas y el cuadro catarral. La forma de presentación más frecuente es como una tumoración cervical asintomática en el ángulo mandibular, 3,6,11,12. Menos frecuentemente puede debutar como masa que deforma la faringe. Así, a veces, se presenta como molestias o leve sensación dolorosa difusa de faringe o como disfagia. En nuestro paciente su forma de presentación fue como una leve odinofagia. Para el paciente la tumoración cervical pasó desapercibida y sólo en la exploración médica es cuando se palpa la misma y se visualiza la masa protuyendo en faringe. Otras veces, puede presentar soplo a la auscultación, dada su rica vascularización, dato que se objetivó en este caso. También puede debutar con disfonía, o parálisis de pares craneales, y así más del $50 \%$ de los pacientes tienen una o más neuropatías craneales al diagnóstico ${ }^{17,19}$. El nervio más comúnmente afectado es el vago seguido por el hipogloso, espinal, glosofaríngeo y facial ${ }^{11,17}$. La presentación como síndrome de Homer, síndrome del agujero yugular, hipoacusia o síncope, también se han descrito. La afectación de una cuerda vocal puede ser tardía, ya que las fibras nerviosas se van distribuyendo alrededor de la tumoración y la compresión de las mismas es gradual, por lo que el cambio de voz puede ser mínimo e inapreciable, aunque en algunas series, la parálisis de la cuerda vocal ipsolateral es, en un $50 \%$ de los casos, el primer signo de presentación.

Pese a que todos los glomus vagales contienen precursores de adrenalina y noradrenalina, hay un reducido número de casos publicados de tumores funcionantes. La producción de catecolaminas por los paragangliomas no está bien documentada y se suele ver más frecuentemente asociada con la presencia de tumores retroperitoneales. Estos dan lugar a un síndrome "feocromocitoma-like" con episodios hipertensivos, rubefacción facial y taquiarritmia. En el caso que se presenta, el paciente es hipertenso mal controlado, a pesar de la medicación, con frecuentes episodios agudos de taquicardia acompañada de sudoración y tras la resección de la tumoración no ha presentado más episodios de crisis hipertensivas en los 3 años que siguen a la intervención quirúrgica ${ }^{6,7,9,12,15,17}$.

Estos tumores tienen posibilidad de malignizarse hasta en un $10 \%$, considerándose el paraganglioma vagal, entre los de cabeza y cuello, el que mayor propensión presenta. La diseminación tumoral se produce tanto vía linfática como hematógena, siendo el pulmón, hueso, riñón y el cerebro los lugares más frecuentes de asiento de metás$\operatorname{tasis}^{2,16,17}$. La presencia de atipias, invasión capsular, actividad mitótica aumentada, invasión neural o vascular no guarda relación con el curso clínico de la enfermedad ni con la malignización ${ }^{1,2,16,17}$. Cuando se llega al diagnóstico de malignidad, en aproximadamente el $65 \%$ de los casos es por la presencia de adenopatías cervicales metastásicas, en $25 \%$ por metástasis a distancia en los lugares antes referidos y en un $10 \%$ por ambas ${ }^{17}$. Las formas malignas son más frecuentes en los casos de paragangliomas funcionantes ${ }^{16}$.

La multicentricidad en los paragangliomas de cabeza y cuello es en general del $10 \%$, siendo de un $5 \%$ para los vagales esporádicos, y elevándose hasta un 33-35\% en los casos familiares, llegando incluso en algunas series hasta el 90\%. En más de un 90\% de los casos de multicentricidad uno de los tumores es carotídeo. Se han descrito casos infrecuentes donde el glomus vagal es bilateral ${ }^{12,17}$.

Los paragangliomas vagales tienen una incidencia familiar de aproximadamente un $8 \%$, siendo el patrón de herencia autosómico dominante, con un grado variable de penetración, por lo que es recomendable el "screening" familiar cuando se detecta algún paciente con esta patología $^{17}$. El paciente del estudio no presenta ni diseminación linfática ni otros paragangliomas en otra localización y en la revisión de su historia familiar no se encuentra ningún antecedente de paragangliomas.

La TC o la RMN con contraste son las técnicas diagnósticas iniciales indicadas, ya que describen la localización anatómica de la lesión. El glomus vagal, por su localización en el compartimento postestiloideo del espacio parafaríngeo, desplaza los vasos anterior y medialmente ${ }^{20}$, a diferencia del carotídeo que ensancha la bifurcación carotídea, y se sitúa generalmente más craneal respecto a los paragangliomas del cuerpo carotídeo. Como todos los paragangliomas muestra la típica imagen en "sal y pimienta" en la RMN en T1, por la hipervascularización de la lesión. La "sal" o áreas de alta señal dentro del parénquima tumoral se corresponden con zonas donde se han producido focos de hemorragia subaguda. La "pimienta" o áreas de baja señal se deben a los vasos arteriales nutricios de la tumoración, que poseen flujo de alta velocidad. La información que aporta la RNM es similar a la obtenida mediante la TC, aunque determina mejor la relación tumoral con los vasos sanguíneos cervicales, por lo que está especialmente indicada para el rastreo de lesiones tumorales preclínicas en familiares ${ }^{3,12,14,20}$.

La angiografía muestra la vascularización del tumor y permite el diagnóstico diferencial con otras lesiones, demostrando el característico desplazamiento anterior de ambas carótidas. Se visualiza agrandamiento de los vasos nutricios tumorales con un prolongado e intenso teñido vascular, y un rápido drenaje venoso secundario a los "shunts" arteriovenosos. En este caso el vaso nutricio es la arteria faríngea ascendente, como suele ser lo habitual. Esta técnica nos permite, además de llegar al diagnóstico, la búsqueda de lesiones multicéntricas, evaluar la circulación colateral al cerebro, y la realización de una embolización selectiva del glomus. Existen opiniones contrapuestas sobre el uso de la embolización prequirúrgica, ya que 
si bien disminuye el tamaño tumoral y el sangrado en la intervención, conlleva el riesgo de provocar un accidente vascular cerebral con posibles secuelas neurológicas. Las lesiones glómicas vagales más próximas al agujero yugular tienen mayor tendencia al sangrado que aquéllas situadas más inferiormente en la región cervical. La punción aspirativa con aguja fina no añade información adicional y al ser una lesión altamente vascularizada, puede suponer un riesgo posterior de sangrado, tras la realización de esta técnica diagnóstica ${ }^{2,11,13,17,18,20}$.

La evaluación de pacientes con paragangliomas vagales familiares puede realizarse también con el uso de la escintigrafía con indio pentetreótide. Se inyecta el contraste seguido de una serie de imágenes radiográficas, lo que permite el diagnóstico de las lesiones cuando aún son de pequeño tamaño, con lo que su manejo es mas fáci $1^{14,17}$.

Anatomopatológicamente se observan nidos celulares característicos llamados "zellballen" y compuestos por “células chief" y "células de sostén" rodeadas por un estroma fibromuscular. Al microscopio electrónico se observan grandes acúmulos de gránulos neurosecretores intracelulares.

Las principales entidades con las que debemos realizar diagnóstico diferencial, por la localización donde asientan, son los schwannoma vagales, meningiomas del espacio carotídeo, y paragangliomas yugulares o carotídeos. Los schwannomas son masas fusiformes en el espacio carotídeo pero angiográficamente no presentan arterias nutricias aumentadas de tamaño ni teñido tumoral. Los meningiomas en estas localizaciones se extienden desde el agujero yugular, el cual muestra cambios óseos escleróticos y de mayor porosidad. El teñido tumoral durante la angiografía es más tenue que en los paragangliomas. Finalmente, se debe diferenciar de los paragangliomas glómicos donde hay un aumento de la horquilla carotídea y de los yugulotimpánicos donde la masa se centra en el agujero yugular cuyos bordes óseos son permeables ${ }^{3,14,20}$.

El tratamiento curativo tanto de los paragangliomas benignos como de los malignizados es la extirpación quirúrgicaa ${ }^{4,12-14,17}$. Por tratarse de tumores benignos de lento crecimiento, aproximadamente $1 \mathrm{~mm}$ de media por año, su tratamiento sigue siendo controvertido. En pacientes sin sintomatología o de edad avanzada parece lógica la abstención terapéutica. En pacientes más jóvenes, en los que, por su localización, puede dar lugar a una morbilidad en fases avanzadas de su evolución o casos sintomáticos, la cirugía en el tratamiento de elección. La indicación quirúrgica debe ser seleccionada cuidadosamente en cada paciente, habida cuenta la morbilidad potencial derivada de la lesión de estructuras vasculares y nerviosas. Previamente a la cirugía se debe haber realizado un buen estudio de la situación tumoral, la resecabilidad y la relación con estructuras vecinas, sobre todo valorando aquéllas que pueden estar englobadas dentro del tumor y que pueden, por tanto, ser sacrificadas en la cirugía, prestando especial atención a los grandes vasos cervicales. Si se presupone la necesidad de resección de la arteria carótida interna es recomendable la realización prequirúrgica del test de oclusión con balón para determinar la viabilidad de la circulación cerebral colateral. En caso de ser necesaria la extirpación de algún vaso o nervio estos pueden reconstruirse posteriormente con injertos. En este paciente no están involucrados ni los. grandes vasos arteriales y venosos cervicales ni otros pares craneales aparte del vago.

Gracias a los avances en las técnicas quirúrgicas, la radioterapia se ha visto desplazada como tratamiento de base, pese a presentar buenos resultados en el control local del tumor. Para algunos autores este tumor no es radiosensible, ya que no existe efecto de destrucción celular directo, sin embargo, sí se conoce que la radioterapia induce la formación de fibrosis y produce disminución de la vascularización de la tumoración consecutiva a una endarteritis obliterativa. Estos cambios fibróticos complican una posible cirugía posterior $\mathrm{y}$, a largo plazo, pueden inducir la malignización de la lesión. Dado que el comportamiento tumoral tras la irradiación es impredecible, debería reservarse para personas mayores, pacientes con lesiones irresecables, tumores residuales tras la cirugía y para aquéllos que, por su estado de salud, no sean subsidiarios de cirugía, con el objetivo de enlentecer el crecimiento local del tumor ${ }^{14}$. En el manejo de los paragangliomas cervicales, como sucede en nuestro caso, la radioterapia no se considera como tratamiento de primera línea, ya que los tumores en esta localización son accesibles para una extirpación quirúrgica completa ${ }^{10}$. En el caso de lesiones bilaterales, una de las lesiones se extirpa, generalmente la de mayor tamaño y la contralateral se trata con radioterapia o simplemente se mantiene una actitud expectante con revisiones periódicas ${ }^{5,12}$.

En nuestro caso se realizó la extirpación tumoral completa con sección del nervio vago, y no se le administró radioterapia postoperatoria. Tras la intervención quirúrgica se produjo parálisis de la cuerda vocal ipsolateral. La parálisis de cuerda vocal unilateral predispone a aspiraciones así como a cambios de voz, especialmente si la cuerda era móvil antes de la intervención quirúrgica. En cualquier caso, después de la cirugía, hay parálisis de la cuerda vocal, ya que se sacrifica el nervio vago, en la mayoría de los casos $^{11,17}$. Otro fenómeno que se puede producir tras la lesión del vago es la parálisis del paladar blando ipsolateral, con la posible regurgitación de líquidos y la aparición de voz de características nasales. Por tanto, el sacrificio del nervio vago es la mayor morbilidad asociada con la resección de glomus vagales, y produce cambios de voz y aspiraciones en mayor o menor grado ${ }^{4,19}$.

La lesión de los nervios glosofaríngeo e hipogloso es relativamente frecuente, encontrándose series en la literatura de aproximadamente un $30 \%$ de afectación. Sin 
embargo, aquí se lograron preservar. Otras posibles complicaciones descritas, pero que tampoco se han presentado, son las lesiones de los vasos sanguíneos adyacentes a la masa tumoral y los problemas en las vías aéreas justificados por la propia intervención quirúrgica. En la intervención se produjo una pérdida sanguínea aproximada de $490 \mathrm{ml}$ similar a las cifras medias de otras series ${ }^{13}$, justificándose este menor sangrado por la realización de una embolización previa a la extirpación quirúrgica. Los paragangliomas vagales, a diferencia de los carotídeos, se suelen localizar más cranealmente y no están tan íntimamente relacionados con los grandes vasos por lo que las posibilidades de lesionarlos y la pérdida sanguínea son menores ${ }^{14,17}$.

Para el tratamiento de las secuelas de la parálisis de una cuerda vocal, se han descrito diversos tratamiento que incluyen la inyección de teflón en la cuerda vocal, medialización de la cuerda vocal paralizada, la miotomía cricofaríngea o la cirugía de la insuficiencia velofaríngea, que pueden ser necesarios en un segundo tiempo. Este paciente ha sido tratado por un logopeda que le ha instruido en técnicas adecuadas de fonación, por lo que no ha requerido cirugía posterior para el tratamiento de las secuelas ${ }^{7,14,17}$.

Hoy en día, a pesar de los abordajes cervicales y de base del cráneo, la embolización y la microcirugía, la cirugía de los paragangliomas vagales tiene un índice de recurrencia del $17 \%{ }^{14}$. En este caso han transcurrido 3 años de seguimiento, sin que se haya objetivado ninguna otra masa de similares características.

\section{Bibliografía}

1. Cakmaksi, H., Usal, C., Guneri, A.: Metastatic hipervascular lynph nodes in malignant glomus vagale tumor: angiography findings. Clin Imaging 2001; 25: 167-170.

2. Carlsen, C.S., Godballe, C., Krogdahl, A.S., Edal, AL.: Malignant vagal paraganglioma: report of a case treated with embolization and surgery. Auris Nasus Larynx. 2003; 30: 443446.

3. Díaz Manzano, J.A., Medina Banegas, A., Osete Albaladejo, J.M., López Meseguer, E.: Paraganglioma of the vagus. A case report and review of the literatura. An Otorrinolaringol Ibero Am. 2003; 30: 127-136.

4. Díez Porres, L., García Iglesias, F., Pérez Martín, G., García Puig, J., Gil Aguado, A.: Multiple paraganglioma: careful with surgery. Rev Clin Esp. 2003; 203: 434-438.

5. Evenson, L.J., Mendenhall, W.M., Parsons, J.T., Cassisi, N.J.: Radiotherapy in the management of chemodectomas of the carotid body and glomus vagale. Head Neck 1998; 20 : 609-613.

6. Groblewsky, J.C., Thedki, A., Carrau, R.L.: Secreting vagal paraganglioma. Am J Otolaryngol 2004; 25: 295- 300.

7. Guiral, H., Risco, J.. García, B., Mayayos, E.: Functioning glomus vagale tumor: report of a case. J Oral Maxillofac.
Surg. 1996; 54: 227-230.

8. Jansen, J.C., Van der Berg, R., Kuiper, A., et al.: Estimation of growth rate in patients with head and neck paragangliomas influences the treatment proposal. Cancer 2000; 88: 2811-2816.

9. Levin, R.J., Hamill, N.J., Grenko, R.T., Huang, M.Y., Fedok, F.G.: Dopamine-secreting glomus vagale: a case report and histopathologic correlation. Head Neck 1998; 20: 753-757.

10. Mendenhall, W.M., Hinerman, R.W., Amdur, R.J., Stringer, S.P., Antonelli, P.J., Singleton, G.T., Cassisi, N.J.: Treatment of Paragangliomas with Radiation Therapy. Paragangliomas of the Head and Neck. The Otolaryngologic Clinics of North America Oct 2001; 34: 1007-1020.

11. Netterville, J.L., Jackson, C.G., Miller, F.R., et al.: Vagal paraganglioma : a review of 46 patients treated during a 20-year period. Arch Otolaryngol Head Neck Surg 1998; 124 : 1133-1140.

12. Pellitteri, P.K., Rinaldo, A., Myssiorek, D., Jackson, G.C., Bradley, P.J., Devaney, K.O., Shaha, A.R. et al.: Paragangliomas of the Head and Neck. Oral Oncology. 2004; 40: 563-575.

13. Persky, M.S., Setton, A., Niimi, Y., Hartman, J., Frank, D., Berenstein, A.: Combined endovascular and surgical treatment of head and neck paragangliomas - a team approach. Head Neck 2002; 24: 423-431.

14. Rao, A.B., Koeller, K.K., Adair, C.F.: Paragangliomas of the Head and Neck: Radiologic-Pathologic Correlation. Radiographics 1999; 19: 1605-1632.

15. Riley, N., Allison, R.: A glomus vagale tumour presenting with acute left ventricular failure. J Laryngol Otol 2003;117: 660-661.

16. Rinaldo, A., Ferlito, A., Myssiorek, D., Devaney, K.O.: Which paragangliomas of the head and neck have a higher rate of malignancy? Oral Oncology 2004; 40: 458-460.

17. Sniezek, J.C., Netterville, J.L., Sabri, A.N.: Vagal, paragangliomas in Paragangliomas of the head and neck. Otolaryngol Clin North Am 2001; 925-939.

18. Tasar, M., Yetiser, S.: Glomus tumors: therapeutic role of selective embolization. J Craniofac Surg 2004; 15: 497-505.

19. Urquhart, A.C., Johnson, J.T., Myers, E.N., Schechter, G.L.: Glomus vagale: Paraganglioma of the vagus nerve. Laryngoscope 1994; 104: 440-445.

12. Weissman, J.L.: Case 21: glomus vagale tumor. Radiology 2000; 215: 237-242.

Torres-Carranza, E.; Infante-Cossío, P.; García-Perla, A.; Belmonte, R.; Menéndez, J.; Gutiérrez-Pérez, J.L.: Paraganglioma del nervio vago. Neurocirugía 2006; 17 : 255-260.

Correspondencia postal: Dr. Eusebio Torres Carranza. Servicio de Cirugía Maxilofacial. Hospital Universitario "Virgen del Rocío" Avda. Manuel Siurot s/n. 41013 Sevilla (España). 